\title{
Significance of the tethered maxillary frenulum: a questionnaire-based observational cohort study
}

\author{
Sody A. Naimer, $M D^{1,2,3}$, Ariel Israel, $\mathrm{MD}^{4}$, Aviezer Gabbay, $\mathrm{MD}^{5,6}$ \\ ${ }^{1}$ Department of Family Medicine, Siaal Family Medicine and Primary Care Research Center, Faculty of Health Sciences, Ben-Gurion University of the Negev, Beer- \\ Sheva, Israel; ${ }^{2}$ Elon Moreh Clinic, Clalit Health Services - Shomron district, Lev Shomron, Israel; ${ }^{3}$ Community Pediatric Service, Kedumim Family Health Center, \\ Clalit Health Services -Shomron district, Lev Shomron, Israel; ${ }^{4}$ Division of Planning and Strategy, Department of Research of Data, Clalit Health Services, Tel Aviv, \\ Israel; ${ }^{5}$ Department of Family Medicine, Faculty of Health Sciences, Hebrew University of Jerusalem, Jerusalem, Israel; ${ }^{6}$ Family Health Center, Clalit Health Services, \\ Jerusalem district, Jerusalem, Israel
}

Background: The clinical significance of lip-tie, or a tethered maxillary frenulum, remains under debate. Clinicians and parents are often perplexed when deciding whether procedures available to relieve a seemingly tight or severe maxillary frenulum are needed.

Purpose: No previous studies have assessed the consequences of not subjecting a tethered maxillary frenulum in newborns to surgical intervention. This study aimed to contribute the first prospective trial on this topic with a relatively extended followup of these newborn infants.

Methods: This prospective observational questionnaire-based cohort trial was performed in a community setting and aimed to determine whether lip-tie is associated with an increased likelihood of eventual feeding or oral disorders.

Results: The convenience sample comprised of 61 consecutively arriving infants with concomitant tethered frenula who were treated at the clinic for various reasons. This cohort was compared with a random sample of 66 age-matched children for a mean follow-up period of 6.42 years. Infants undergoing oropharyngeal procedures were excluded. Awareness of a deviation in oral structures was reported by $18 \%$ of the study group versus $0 \%$ of the controls. Mothers participating in the study group (24.6\%) less frequently recalled painful nipples or discomfort during breastfeeding than those in the control group $(47.0 \%)(P<0.01)$. There were no intergroup differences in other types of feeding difficulty, dental hygiene, pronunciation, or speech development.

Conclusion: Our findings suggest that a tethered labial frenulum is not associated with an increase in breastfeeding disturbances or oral disorders. These data encourage clinicians to question the need to intervene in cases of tethered maxillary frenula.

Key words: Breastfeeding, Frenulum, Infant welfare, Oral pathology, Tethered maxillary frenulum

\section{Key message}

Question: Does a tethered maxillary frenulum in the newborn result in breastfeeding difficulty or other oral symptomatology?

Finding: The analysis of subjects with a tethered maxillary frenulum surveyed beyond a mean 5-year follow-up did not reveal an increase in oral issues versus those of a random agematched control group.

Meaning: These data demonstrate no need to intervene upon the diagnosis of a tethered maxillary frenulum.

\section{Introduction}

Labial frenal attachments are thin folds of mucous membrane with enclosed muscle fibers that originate from the orbicularis oris muscle of the upper lip attached at the lips to the alveolar mucosa and underlying periosteum. ${ }^{1)}$ Both the labial and lingual frenula have been implicated in issues regarding breastfeeding difficulty. "Lip-tie," an advanced grade of the labial frenulum or tethered maxillary frenulum, is not only considered to be instrumental in a number of disorders, but is also associated with improper latching of the newborn to the mother's breast, thereby leading to painful nipples and increased infant reflux and aerophagia. ${ }^{2,3)}$ The condition has also been implicated as the cause of bottle-feeding difficulties, gingival recession, midline diastema, speech impediments, and even dental caries. ${ }^{4-6)}$ Relatively few studies have focused on the natural history of the maxillary labial frenulum. In a study of children ages 1 to 8 years, Boutsi and Tatakis ${ }^{7)}$ found that attachment of the frenulum differs across ages, with older children showing mucosal or gingival frenula rather than papillary penetrating frenula; this finding suggests that the maxillary labial frenulum may shift its insertion point as a child ages and as the maxilla develops.

In addition, 6 separate research teams reporting on physical inspections of the oral cavities of thousands of newborns chose to completely ignore this structure. ${ }^{1,8-14)}$ The ubiquitous appea-

Corresponding author: Sody Naimer. Department of Family Medicine, Faculty of Health Sciences, Ben-Gurion University of the Negev, P0B 653, Beer-Sheva 84105, Israel 凶Email: sodyna@clalit.org.il, https://orcid.org/0000-0003-2614-147X

Received: 26 March, 2020, Revised: 16 August, 2020, Accepted: 19 August, 2020

This is an open-access article distributed under the terms of the Creative Commons Attribution Non-Commercial License (http://creativecommons.org/licenses/bync/4.0/) which permits unrestricted non-commercial use, distribution, and reproduction in any medium, provided the original work is properly cited.

Copyright (c) 2021 by The Korean Pediatric Society 


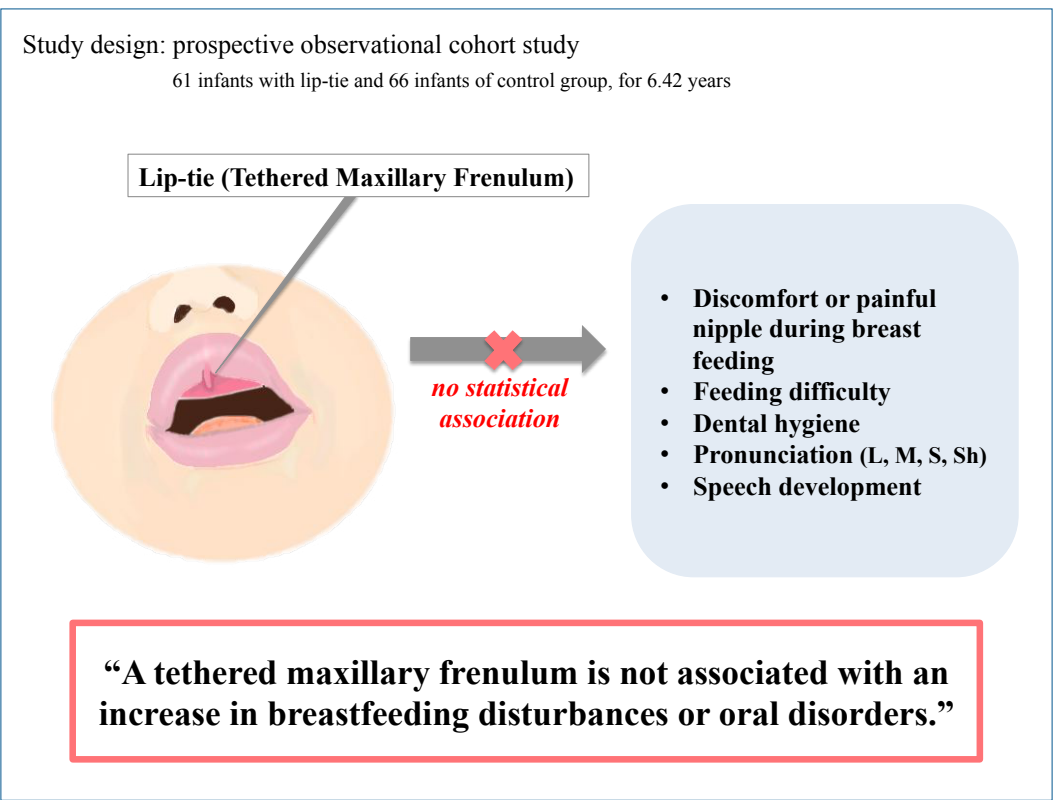

Graphic Abstract.

rance to any extent in infants of labial or maxillary frenal attachments may be part of the reason for this disregard. ${ }^{9-13)}$ Conflicting views concerning the significance of the tethered maxillary frenulum prevail; in fact, aside from expert opinions that are unsubstantiated by research, hardly any data exists on the subject. 15,16) Consistently, publications written about procedures to correct this anatomy avoid any reference to their actual need. ${ }^{17-23)}$ Thus, a solid scientific basis justifying such surgical intervention is necessary to determine if it is at all warranted.

A number of research studies have attempted to classify the spectrum of maxillary frenulum attachments based primarily on point of attachment of the distal frenulum margin.,24-26) Kotlow 2) developed a four-grade system upon which he bases decisions concerning when surgical intervention may benefit a presenting infant. He proposes that the higher the grade of membrane attachment to the gingiva of the pre-maxilla, the greater the association with breastfeeding problems. Despite the claims of Santa Maria et al., who devised a similar classification combining 2 of the grades in order to provide a more meaningful clinical distinction, Kotlow's classification seems to be the most prevalent and widely accepted. ${ }^{25,26)}$

To date, no prospective study has followed over time neonates with the most severe degree of frenulum tethering in order to determine whether it is correlated with the development of breastfeeding difficulty, dental hygiene, or functional problems.

\section{Methods}

This trial was designed to be a prospective observational cohort study. The subjects forming our convenience sample were infants mostly in their first weeks and months of life. A patient was selected for study participation when the physician conducting a routine clinical encounter incidentally identified the infant as presenting with a 'tethered' maxillary frenulum (TMF), defined as a Stanford grade 3 or a grade 3-4 on Kotlow's scale. The current research was incorporated into routine medical care and did not entail an intentional meticulous buccal examination of each visiting child. Likewise, a group of age-matched infants consecutively visiting the clinic for a variety of reasons served as a convenience sample of controls. Patients that could not be contacted upon trial completion as well as those undergoing oropharyngeal surgical procedures were excluded from both the study and control groups.

We expected an increase of breastfeeding difficulties beyond the normally accepted rate (15\%-20\%), while also anticipating that an elevated predominance of oral hygiene and function issues would appear only if these were greatly increased. Therefore, we calculated that the appearance of complications at an incidence greater than threefold (40\%-50\%) would be sufficient to revoke the null hypothesis and demonstrate a significant rise in frequency. Because a power analysis conducted prior to the study estimated a needed sample size of 51 participants to attain a 0.05 alpha level with a power of $80 \%$, we expected a sample size of 55 participants to suffice in addressing the hypothesis of the research. This calculation was derived via the command sampsi in the Stata 12 (StataCorp LP., College Station, TX, USA).

Upon study completion, each participant's family members were contacted, then subsequently questioned about breastfeeding and feeding issues, dentition development, dental hygiene, aesthetic appearance, oral health, and speech function.

Data was retrieved via a structured questionnaire completed by a parent or by a research assistant conducting a personal interview in accordance with the same questionnaire. Data analysis was performed using the SPSS ver. 17 (SPSS Inc., Chicago, IL, USA). Quantitative variables, e.g., age, and categorical variables, e.g., sex and the presence of accompanying illness, were used to describe characteristics of the research population, while quanti- 
Table 1. Comparison of demographic and clinical features of the study population

\begin{tabular}{|c|c|c|c|}
\hline Variable & Study group ( $n=61$ ) & Control group $(n=66)$ & $P$ value \\
\hline Age (yr) & & & 0.381 \\
\hline$<2$ & $0(4.9)$ & $2(3.0)$ & \\
\hline $3-4$ & $5(8.2)$ & $12(18.3)$ & \\
\hline $5-6$ & $28(45.9)$ & $23(34.8)$ & \\
\hline $7-8$ & $13(21.3)$ & $13(19.7)$ & \\
\hline $9-10$ & $12(19.7)$ & $14(21.2)$ & \\
\hline$\geq 11$ & $0(0)$ & $2(3.0)$ & \\
\hline Parents' Origin Israel & $55(90.2)$ & $60(90.9)$ & 0.805 \\
\hline Abroad & $6(9.8)$ & $6(9.1)$ & \\
\hline Asia & $2(3.3)$ & $3(4.5)$ & \\
\hline Africa & $3(4.9)$ & $1(1.5)$ & \\
\hline Europe & $3(4.9)$ & $6(9.1)$ & \\
\hline North America & $2(3.3)$ & $1(1.5)$ & \\
\hline Male sex & $38(62.3)$ & $31(47.0)$ & 0.109 \\
\hline Any anomaly noticed in structure of mouth or gums following birth & $11(18.0)$ & $0(0)$ & $<0.001$ \\
\hline Awareness of accompanying illnesses when diagnosed with finding & $4(6.7)$ & $1(1.5)$ & 0.191 \\
\hline Existence of any family members with tongue or gum impediments & $18(29.5)$ & $8(12.1)$ & 0.833 \\
\hline Existence of Any family members with upper lip-tie & $7(38.9)$ & $3(37.5)$ & 0.164 \\
\hline Existence of any familial congenital defects & $14(23.0)$ & $14(21.2)$ & 0.834 \\
\hline Existence of breastfeeding difficulties (values 0-10) & $2.7 \pm 3.6$ & $2.0 \pm 2.5$ & \\
\hline Any remembered nipple pain during breastfeeding & $15(24.6)$ & 31 & 0.010 \\
\hline The aesthetic appearance of lip-tie was bothersome & $0(0)$ & $0(0)$ & 1.000 \\
\hline
\end{tabular}

Values are presented as number (\%) or mean \pm standard deviation.

Boldface indicates a statistically significant difference with $P<0.05$.

Table 2. Comparison of pronunciation difficulties

\begin{tabular}{|c|c|c|c|c|c|c|}
\hline \multirow{2}{*}{ Pronunciation } & \multicolumn{3}{|c|}{ Did your child ever have a problem? } & \multicolumn{3}{|c|}{ Does your child currently have a problem? } \\
\hline & Control group $(n=66)$ & Study group $(n=61)$ & $P$ value & Control group $(n=66)$ & Study group $(n=61)$ & $P$ value \\
\hline Pronouncing "L" & $4(6.1)$ & $3(4.9)$ & 1.000 & $1(1.5)$ & $2(3.3)$ & 0.608 \\
\hline Pronouncing "M" & $1(1.5)$ & $0(0)$ & 1.000 & $1(1.5)$ & $1(1.6)$ & 1.000 \\
\hline Pronouncing "S" & $5(7.6)$ & $3(4.9)$ & 0.719 & $3(4.5)$ & $1(1.6)$ & 0.620 \\
\hline Pronouncing "sh" & $18(27.3)$ & $19(31.1)$ & 0.698 & $9(13.6)$ & $10(16.4)$ & 0.804 \\
\hline
\end{tabular}

Values are presented as number (\%).

tative variables were expressed as averages and, when distributed normally, as standard deviations. When distributed abnormally, quantitative variables were expressed through medians, minimums, and maximums. Categorical variables were expressed as percentages. A comparison between quantitative variables distributed normally was performed by means of a t test, while comparing between quantitative variables distributed abnormally was performed through a Mann-Whitney test. In addition, a comparison between categorical variables was achieved using Fisher exact test; Barnard test was performed, when necessary, to confirm nonsignificance. For all tests the $P$ value of $<0.05$ was considered statistically significant.

\section{Results}

The research cohort comprised a convenience sample of 71 infants who were treated for various reasons at our clinic bet- ween the years 2008-2014 and who presented with the most advanced form of maxillary frenulum. Upon conclusion of the follow-up period lasting through the end of 2018, cumulative data was collected and interpreted. Ten of the 71 children originally recruited for the study were excluded from the prospective follow-up, 4 of them because they had undergone oropharyngeal procedures to eliminate the TMF. In these 4 cases, the decisions to surgically intervene were unrelated to symptoms, but were made to prevent potential future suffering. Additionally, 6 subjects were excluded because they could either not be located or parental consent could not be obtained. Hence, 61 infants were included in the study group and 66 infants were included in the control group. The sociodemographic and clinical parameters of both of these groups were similar, as can be seen in Table 1. Fifty-five infants (90.2\%) of the study group and $60(90.9 \%)$ of controls were Israeli-born $(P=0.805)$. At time of recruitment, $63.9 \%$ were younger than 4 months old, and follow-up continued for an average of 6.42 years. There was no 


\begin{tabular}{|c|c|c|c|}
\hline Dental problems and treatments & Study group $(\mathrm{n}=61)$ & Control group $(n=66)$ & $P$ value \\
\hline Are you aware of a gap between the child's front teeth? & $17(27.9)$ & $14(21.2)$ & 0.414 \\
\hline To what extent does the child suffer from tooth decay & $0.9 \pm 1.7$ & $1.4 \pm 1.6$ & 0.585 \\
\hline To what extent does the child suffer from stained teeth? & $0.9 \pm 1.7$ & $1.4 \pm 1.6$ & 0.105 \\
\hline How often does the child brush teeth daily? & $1.4 \pm 0.6$ & $1.3 \pm 0.7$ & 0.422 \\
\hline 0 & $2(3,3)$ & $8(12.1)$ & 0.163 \\
\hline 1 & $35(57.4)$ & $32(48.5)$ & \\
\hline 2 & 24(39.3) & $26(39.4)$ & \\
\hline How frequent are the child's visits to the dentist? & & & 0.130 \\
\hline Less than every 2 years & $11(18.0)$ & $21(31.8)$ & \\
\hline Once a year or two & $31(50.8)$ & $32(48.5)$ & \\
\hline More than once a year & $19(31.1)$ & $13(19.7)$ & \\
\hline Has the child been treated by the dentist in the past 2 years? & $37(60.7)$ & $(57.6)$ & 0.857 \\
\hline Has the child had dental fillings in the past 2 years? & $26(42.6)$ & $30(45.5)$ & 0.858 \\
\hline Has the child undergone dental extractions in the past 2 years? & $5(8.2)$ & $7(6)$ & 0.765 \\
\hline
\end{tabular}

Table 4. Comparison of the children's clinical status at study completion

\begin{tabular}{|c|c|c|c|}
\hline Clinical status & Study group $(n=61)$ & Control group $(n=66)$ & $P$ value \\
\hline Are you currently aware of any anomalies in oral or dental structure? & $5(8.2)$ & $8(12.1)$ & 0.564 \\
\hline Do you know of any accompanying illnesses currently & $2(3.3)$ & $1(1.5)$ & 0.605 \\
\hline Current accompanying illnesses & & & 0.377 \\
\hline Ear and throat infections & $1(50)$ & $0(0)$ & \\
\hline Periodontal infections & $0(0)$ & $1(100)$ & \\
\hline Nasal speech + developmental lag of 1 year & $1(50)$ & $0(0)$ & \\
\hline $\begin{array}{l}\text { Has anyone ever tried to recommend or influence you to pursue } \\
\text { treatment for this finding during infancy? }\end{array}$ & $7(11.5)^{a)}$ & $1(5.0)^{b)}$ & 0.672 \\
\hline
\end{tabular}

statistically significant difference between the ages of each of the participants in the study and control groups (95.1\% and 93.9\%, respectively, were between the ages of 3 and 8 years). Nor were there gender differences between the children $(P=0.109)$, yet a number of observed clinical differences are worthy of note. Whereas in the study group 11 of the 61 parents (18\%) reported awareness of deviation or abnormality in structures of their childrens' mouths or gums immediately at birth or in the ensuing days $(P<0.0001)$, none of the control group parents reported any such awareness. Furthermore, although $29.5 \%$ of the parents of the study group versus $12.1 \%$ of controls reported they had knowledge of other family members with tongue or gingival abnormalities $(P=0.017)$, even in these cases the abnormalities reported by family members did not entail an increase in frequency of a TMF (38.9\% vs. $37.5 \%$, respectively) $(P=0.053)$. More mothers from the control group recalled painful nipples or other discomfort while breastfeeding (47.0\%) in comparison to the study group $(24.6 \%)(P=0.010)$. Among all other clinical variables, no differences were found between the groups (Table 2).

With regard to pronunciation difficulties or speech development issues, no differences were identified between the 2 groups (Table 3). Likewise, no statistical differences concerning dental condition emerged to differentiate between the groups (Table 4).
Moreover, $11.5 \%$ of the study group as opposed to $5 \%$ of the control group reported past attempts on the part of various individuals to convince them that an intervention was necessary to "treat" their child's maxillary frenulum $(P=0.672)$.

\section{Discussion}

In the distant past, Taylor, upon reviewing the topic of our research question, reflected in his summary that the sheer amount of literature on the subject of surgical intervention as concerns maxillary frena would doubtlessly support the view that this controversial issue is ongoing. However, an alternative viewpoint posits that no surgical intervention poses no problem. Those subscribing to this viewpoint can be categorized into 2 groups. The first group insists that the frenum is abnormal, consequently it should be surgically removed, resolving the simple problem. Conversely, the second group asserts emphatically that there is no problem to begin with, since the frenum, regardless of whether it is normal or abnormal, should rarely, if ever, be surgically removed. The very fact that 2 such diametrically opposed opinions concerning the fate of the frenum exist sufficiently proves that the matter continues to pose a major treatment problem. Taylor 's notions, conceived some 80 years ago, unfortunately, 
remain valid to this day, hence the pertinence of the subject matter of our research. ${ }^{27}$

The diverse tethering of the maxillary frenulum, though com. monplace, is a phenomenon s to which significant and deleterious clinical consequences have been attributed. ${ }^{10,11,23-25)}$ The fact that in our cohort over $5 \%$ of presenting subjects who demonstrated no symptoms whatsoever had to be eliminated from the study because they conceded to surgical intervention highlights the validity of the current study's research question.

A previous study conducted in an Israeli hospital's newborn unit found that virtually all 141 infants that were carried to fullterm presented to a certain extent with both labial and lingual frenula. ${ }^{28)}$ The widespread incidence of this phenomenon further emphasizes the relevance of clarifying its ramifications and concerns had by parents and medical staff.

It has been long established that nipple pain constitutes the most common cause of difficulty with breast feeding and is the second most frequently stated reason for discontinuation. ${ }^{29,30)}$ If an infant's tethered maxillary frenulum results in difficulty "latching on" or maintaining an adequate seal of his mouth, we would have expected our cohort to demonstrate an increase in breastfeeding problems. However, this was not the case. The reported reduced incidence of feeding difficulties in our study group remains a mystery and it would be interesting to see if the same results emerge in future studies.

Other reported consequences of TMF include diastema located between the maxillary incisors, labial "catching" between the incisor teeth, food remnants becoming trapped in oral pockets, impaired oral hygiene and healing of wounds, and speech disturbances. ${ }^{31,32)}$ Yet these hindrances were not observed in our study group. Conversely, adversaries of surgical intervention claim that surgical incisions can cause scarring and nodules which themselves may prevent approximation of the incisors, thereby causing diastema. ${ }^{32}$ Despite the robustness of our findings, the limited size of our sample precludes the drawing of any definitive conclusions regarding these issues.

Our literature review disclosed a single, large point-prevalence study of oral health findings in infants. This research included a prospective component lasting only 5 months which failed to report any associated symptoms correlating with a specific frenulum anatomy. ${ }^{33)}$

Recently review containing a meticulous literature search from 1946 sought an established correlation between upper-lip-tie and its most common proclaimed complication "breastfeeding difficulty" was published at the same time our study neared completion. ${ }^{34)}$ Not a single randomized control trial was identified that showed evidence favoring routine frenulum release in infants with breastfeeding difficulties. The author's concluded that the"... classification system proposed by Kotlow has not been found reliable both in terms of inter and intraobserver agreement and in terms of predicting the severity of the breastfeeding difficulties." Another exciting new publication suggests that a more meaningful assessment of the magnitude of frenulum tethering would necessitate a compilation of parameters including distance from alveolar ridge, width of attachment, length of free lip, and flexibility of frenulum, not the location of frenulum insertion alone. ${ }^{35)}$ It is of further interest to note that, in a study by Dollberg et al., ${ }^{28)}$ follow-up interviews 2 weeks following birth found no association between the most severe cases of "tongue" or "lip-tie" with mothers who choose to cease breastfeeding due to difficulties.

Our preliminary research constitutes the first relatively extended, follow-up study done on infants with a TMF. Our surveillance period covered the complete critical period after birth when breastfeeding issues arise and, for most of the study group, also included a significant period of development when dentition and speech problems may appear.

Limitations of this work include the possibility of a broad variability of frenulum classification, questioned previously. ${ }^{26}$ However, infants' families that receive recommendations to undergo surgical intervention for TMF base their decisions purely upon these same clinical visual criteria. The small number of study participants recruited per month supports our premise that only those with extreme forms of the condition were selected. Information derived included not only current oral health issues, but also details from years prior, relating to breastfeeding disturbance; this time-lapse may give rise to recall bias. The acceptance of parent perception as indication of speech difficulties, as well as reporting of dental hygiene instead of an objective assessment and examination reduces reliability of this data. Finally, an ideal design would dictate examining every single infant who arrives to the clinic for treatment, then comparing study and control groups according to presenting anatomy. We feel such a study would contribute greatly to the field and hope to initiate one in the future. Despite these study limitations, the trend we observed regardless reveals meaningful information that will assist us in consulting patients about the pros and cons of surgical intervention.

In addition to demonstrating the null hypothesis assessed against age-matched comparisons, we showed that infants presenting with a TMF do not suffer consequences, or up to $50 \%$ incidence of disrupted lactation more frequently than does the general population. ${ }^{36}$ ) We recommend considering frenulum release exclusively for infants presenting with ongoing symptoms such as breastfeeding difficulty. It is hoped that this preliminary data will encourage greater interest in this important field and inspire larger studies with longer follow-up periods in diverse populations across the globe.

In conclusion, in our modest attempt to illuminate the significance of the elusive congenital buccal soft tissues, we suggest that tethered labial frenula are not associated with increased breastfeeding disturbances and oral development or function. The data presented here calls into question the need to intervene in this condition.

\section{Conflicts of interest}

No potential conflict of interest relevant to this article was reported. 


\section{References}

1. George D, Bhat SS, Hegde SK. Oral findings in newborn children in and around Mangalore, Karnataka State, India. Med Princ Pract 2008;17:3859.

2. Kotlow LA. Diagnosing and understanding the maxillary lip-tie (superior labial, the maxillary labial frenum) as it relates to breastfeeding. J Hum Lact 2013;29:458-64.

3. Kotlow LA. The influence of the maxillary frenum on the development and pattern of dental caries on anterior teeth in breastfeeding infants: prevention, diagnosis, and treatment. J Hum Lact 2010;26:304-8.

4. Chang C. Upper lip tie and its treatment [Internet]. Warrenton (VA): Fauquier ENT Consultants; 2004-2021 [cited 2018 Jul 8]. Available from: www.fauquierent.net/upperliptie.htm.

5. Kotlow L. Infant reflux and aerophagia associated with the maxillary lip-tie and ankyloglossia (tongue-tie). Clin Lactation 2011;2:25-9.

6. Ceremello PJ. The superior labial frenum and the midline diastema and their relation to growth and development of the oral structures. Am J Orthod 1953;39:120-39.

7. Boutsi EA, Tatakis DN. Maxillary labial frenum attachment in children. Int J Paediatr Dent 2011;21:284-8.

8. Jorgenson RJ, Shapiro SD, Salinas CF, Levin LS. Intraoral findings and anomalies in neonates. Pediatrics 1982;69:577-82.

9. Huang WJ, Creath CJ. The midline diastema: a review of its etiology and treatment. Pediatr Dent 1995;17:171-9.

10. Freudenberger S, Santos Díaz MA, Bravo JM, Sedano HO. Intraoral findings and other developmental conditions in Mexican neonates. J Dent Child (Chic) 2008;75:280-6.

11. Cetinkaya M, OzFT, Orhan AI, Orhan K, Karabulut B, Can-Karabulut DC, et al. Prevalence of oral abnormalities in a Turkish newborn population. Int Dent J 2011;61:90-100.

12. Perez-Aguirre B, Soto-Barreras U, Loyola-Rodriguez JP, Reyes-Macias JF, Santos-Diaz MA, Loyola-Leyva A, et al. Oral findings and its association with prenatal and perinatal factors in newborns and its association with prenatal and perinatal factors in newborns. Korean J Pediatr 2018;61:27984.

13. Liu MH, Huang WH. Oral abnormalities in Taiwanese newborns. J Dent Child (Chic) 2004;71:118-20.

14. Friend GW, Harris EF, Mincer HH, Fong TL, Carruth KR. Oral anomalies in the neonate, by race and gender, in an urban setting. Pediatr Dent 1990; 12:157-61.

15. Lawrence A. Kotlow DDS. Oral diagnosis of abnormal frenum attachments in neonates and infants: evaluation and treatment of the maxillary and lingual frenum using the erbium: YAG laser. Pediatric Dental Care 2004;10 (3).

16. Medeiros Júnior R, Gueiros LA, Silva IH, de Albuquerque Carvalho A, Leão JC. Labial frenectomy with Nd:YAG laser and conventional surgery: a comparative study. Lasers Med Sci 2015;30:851-6.

17. Francis DO, Chinnadurai S, Morad A, Epstein RA, Kohanim S, Krishnaswami S, et al. Treatments for ankyloglossia and ankyloglossia with comcomitant lip-tie. Rockville (MD): Agency for Healthcare Research and Quality (US), 2015. Report No.: 15-EHC011-EF.

18. Kotlow L. Oral diagnosis of abnormal frenum attachments in neonates and infants: evaluation and treatment of the maxillary and lingual frenum using the erbium:YAG laser. J Pediatr Dent Care 2004;10:11-14.

19. Gontijo I, Navarro RS, Naypek P, Ciamponi AL, Haddad AE. The application of diode and Er:YAG lasers in labial frenectomies in infants. J Dent Child 2005;72:10-55.
20. Olivi G, Chaumanet G, Genovese MD, Beneduce C, Andreana S. The Er,Cr:YSGG laser labial frenectomy: a clinical retrospective evaluation of 156 consecutive cases. Gen Dent 2010;58:126-33.

21. Shetty K, Trajtenberg C, Patel C, Streckfus C. Maxillary frenectomy which was done by using a carbon dioxide laser in a pediatric patient: a case report. Gen Dent 2008;56:60-3.

22. Kafas P, Stavrianos C, Jerjes W, Upile T, Vourvachis M, Theodoridis M, et al. Upper-lip laser frenectomy without infiltrated anaesthesia in a paediatric patient: a case report. Cases J 2009;2:7138.

23. Hansen J. Doctors warning parents to stop new fad of operating on their baby's tongues. The Sunday Telegraph. 2016 March 27. Available from: www.dailytelegraph.com.au/news/doctors-warning-parents to-stop-newfad-of-operating-on-their-baby- tongues/newsstory/bdc5a7fe78e74da01b 3290d85ab14655.

24. Placek M, Skach M, Mrklas L. Problems of the labial frenum attachment in periodontics. II. Attempt to determine the resistance of periodontium to the influence of individual types of the labial frenum attachment. Cesk Stomatol 1974;74:401-6. Czech.

25. Delli K, Livas C, Sculean A, Katsaros C, Bornstein MM. Facts and myths regarding the maxillary midline frenum and its treatment: a systematic review of the literature. Quintessence Int 2013;44:177-87.

26. Santa Maria C, Aby J, Truong MT, Thakur Y, Rea S, Messner A. The superior labial frenulum in newborns: what is normal? Global pediatric health. 2017;4:1-6.

27. Taylor JE. Clinical observations relating to the normal and abnormal frenum labii superioris. Am J Orthod Oral Surg 1939;25:646-50.

28. Dollberg S, Haham A, Botzer E, Golan C. Prevalence and descriptive anatomy of lingual and labial frenula in newborn infants and their relation to breastfeeding difficulties. In: Poster presented at: Annual Conference of Israel Society of Clinical Pediatrics; 2014; Tel Aviv, Israel.

29. Martin J, Monk J. Infant feeding practice in 1980. London: Office of Population Censuses and Surveys, 1983.

30. Sloper KS, Elsden E, Baum JD. Increasing breast feeding in a community. Arch Dis Child 1977;52:700-2.

31. Minsk L. The frenectomy as an adjunct to periodontal treatment. Compend Contin Educ Dent 2002;23:424-6.

32. Edwards JG. A clinical study: the diastema, the frenum, the frenectomy. Oral Health 1977;67:51-62.

33. Flinck A1, Paludan A, Matsson L, Holm AK, Axelsson I. Oral findings in a group of newborn Swedish children. Int J Paediatr Dent 1994;4:67-73.

34. Nakhash R, Wasserteil N, Mimouni FB, Kasirer YM, Hammerman C, BinNun A. Upper lip tie and breastfeeding: a systematic review. Breastfeed Med 2019;14:83-7.

35. Ray S, Golden WC, Walsh J. Anatomic distribution of the morphologic variation of the upper lip frenulum among healthy newborns. JAMA Otolaryngol Head Neck Surg 2019;145:931-8.

36. Stuebe A, Horton BJ, Chetwynd E, Watkins S, Grewen K, Meltzer-Brody S. Prevalence and risk factors for early, undesired weaning attributed to lactation dysfunction. J Womens Health (Larchmt) 2014;23:404-12.

How to cite this article: Naimer SA, Israel A, Gabbay A. Significance of the tethered maxillary frenulum: a questionnairebased observational cohort study. Clin Exp Pediatr 2021;64: 130-5. https://doi.org/10.3345/cep.2020.00486 